\title{
Observaçōes preliminares sobre a transmissão de oncocercose no rio Toototobi, Amazonas, Brasil
}

A. J. Shelley ( $\left.{ }^{*}\right)$

J. A. S. Nunes de Mello $\left({ }^{* *}\right)$

R. G. O. Rees (")

\section{Resumo}

Em dezembro de 1975 os autores estudaram vários fatores que influenciam a transmissão de Onchocerca volvulus ao norte do Amazonas, no rio Toototobi. Observaram que a densidade de micro. filárias de $\mathbf{O}$. volvulus era maior no tronco do que nos membros de dois índios Yanomama examinados. Foram capturados três espécies antropófilas de Simuliidae, provisoriamente denominados Simu. lium sp. A, S.sp.B e S.sp.C. A primeira espécie foi coletada em grande número no começo da tarcle. atacando o homem. O encontro de larvas salsichoides em espécimes naturalmente infetados e o sucesso da infecção experimental de espécimes selvagens com microfilárias de $\mathbf{O}$. volvulus que desenvolveram até o final do estágio salsichóide, sugerem que o provável vetor de oncocercose nesta área é o Simulium sp. A.

\section{INTRODUÇÃo}

Bearsoti et al. (1967) foram os primeiros a assinalar a presença de oncocercose no Brasil. Mais tarde, Moraes \& Dias (1972), Moraes et al. (1973), e Moraes \& Chaves (1974a, b) demonstraram a existência desta doença, principalmente entre os índios Yanomama, no norte do Brasil. Rassi (1974) e Rassi et al. (1975) referem-se à observaçăo de uma nova espécie vetora, Simulium amazonicum, naturalmente infetada por $O$. volvulus às margens do rio Toototobi, no Amazonas.

Em dezembro de 1975 os autores fizeram uma viagem de dez dias à missão Toototobi, realizando investigações preliminares sobre a distribuição de microfilárias de $O$. volvulus no corpo humano. Também foram feitas coletas de simulídeos antropófilos para estudar seus hábitos e testar seu desempenho como vetores em potencial de oncocercose.

\section{MATERIAL E MÉTODOS}

Foi feita biópsia de pele dos ombros e nádegas de sete índios; o material foi tratado segundo a técnica de Duke (1962), e as lâminas coradas com hematoxilina de Mayer. Por falta de condiçōes não foi possivel proceder a pesagem dos fragmentos de pele. Dois destes índios, Roberto e Raimundo, ambos com cerca de 40 anos, foram selecionados para os experimentos de distribuição de microflárias de $O$. volvulus na pele, por apresentarem na amostra as maiores densidades destas micro. filárias. Estes índios não apresentaram microfilárias de Mansonella sp. nas biópsias de pele, nem em esfregaçus de sangue periférico. Procedeu-se à avaliação da distribuição das microfilárias na pele dos voluntários pela retirada de fragmentos de tamanhos aproximadamente iguais do pescoço, parte superior e inferior das costas, nádegas, peito, abdome. antebraços, braços, coxas e pés de Raimundo. Também foi aplicada em ambos a técnica de Leon \& Duke (1966), que utiliza a ingestão de microfilárias por simulídeos. As coletas de simulideos foram realizadas na varanda de uma casa entre 16,00 e 18,00 horas, hora local, usando-se Roberto como isca durante dois dias consecutivos, e Raimundo um dia apenas. As fêmeas, quando repletas, eram coletadas em frascos e mortas com fumaça de tabaco. O estômago era dissecado e feito esfregaço do repasto sanguíneo que, após desemoglobinização, era corado com hematoxilina de Mayer. Procedia-se então a identificação e a contagem das microfilárias nas preparaçōes.

Para tentar determinar a identidade do vetor de oncocercose no Toototobi foram coletadas fêmeas de simulídeos entre 16,00 e 18,00

(") - Universidade de Brasilia, DF.

(*) - Instituto Nacional de Pesquisas da Amazônia, Manaus. 
horas, usando-se Roberto e Raimundo como iscas durante quatro dias seguidos. As fêmeas ropletas coletadas das diferentes partes do corpo da isca eram guardadas individualmente em tubos de vidro de $5 \times 1 \mathrm{~cm}$ com fundo e boca cobertos respectivamente com discos de papel de filtro e gaze fina. Uma tira estreita de papel de filtro era colocada em posição vertical em cada tubo para servir de repouso aos simulídeos.

Todos os tubos foram guardados inicialmente em um dessecador no qual uma solução saturada de sulfato de amônio mantinha a umidade cerca de $80 \%$. Entretanto a temperatura variou entre 20 e $32^{\circ} \mathrm{C}$, causando condensação nos tubos e provocando uma alta taxa de mortalidade entre os insetos. Nos três dias subseqüentes os tubos foram colocados em uma caixa de isopor contendo papel molhado, obtendo-se assim um ambiente com temperatura entre 19 e $20^{\circ} \mathrm{C}$ e umidade relativa maior que $85 \%$. Um dia após o repasto infectante foram feitas tentativas para que os dipteros aceitassem novas refeições sanguíneas, apesar de ter sido colocado na boca de cada tubo um algodão embebido em solução açucarada a $10 \%$ ou papa de banana. De 12 em 12 horas examinava-se os tubos e todo inseto ericontrado morto era conservado em álcool a $70 \%$, os vivos eram transferidos para tubos limpos e novamente tentado o processo de alimentação. No laboratório os insetos preservados eram deixados em água durante um dia e em seguida cada espécime colocado em uma lâmina e dividido em cabeça, tórax e abdome. Preparados esfregaços com o conteúdo do abdome após dissecação. Quando presente, geralmente era possível macerar o sangue em água com agulhas finas de dissecção. O tegumento abdominal era espalhado uniformemente na lâmina perto do conteúdo do estômago e a preparação deixada secar ao ar. Cabeça e tórax, ligeiramente quebrados para expor a musculatura, eram colocados em outra lâmina. Foi posta uma gota de hematoxilina de Mayer sobre cada porção do corpo do inseto durante 30 a 45 minutos, e peças transferidas em seguida para a água. Cabeça e tórax foram dissecados separadamente em uma gota de glicerina e depois montadas. A lâmina com o esfregaço de sangue e a parede abdominal foi também montada em glicerina. A procura de parasitas foi feita com 200x aumento.

Durante três dias consecutivos observouse a incidência de picadas dos simulídeos, usando-se um índio como isca. Ele permanecia de pé durante todo o dia na varánda da casa, com pequenos intervalos de descanso. As coletas realizadas por dois coletores começaram ao alvorecer $(06,00$ horas) prclongando-se até um pouco depois do anoitecer $(19,00$ horas), registrando-se sempre a hora em que os insetos eram coletados. Os simulídeos capturados foram sacrificados e conservados em álcool para serem examinados mais tarde. Temperatura, umidade relativa, chuva e nebulosidade eram registrados de hora em hora durante estas observações. Mais tarde no laboratório, foram feitas dissecções à procura de infecção com $O$. volvulus, como descrito acima.

\section{Resultados}

Três espécies de simulídeos foram capturados enquanto picavam isca humana. Como não foi possivel ainda uma identificação definitiva os insetos capturados serão referidos como Simulium sp. $A$, sp. $B$, e sp. $C$., sendo a primeira espécie encontrada em grande quantidade.

\section{DISTRIBUIÇÃO DE MICROFILÁRIAS NA PELE}

No quadro I são apresentados os números de microfilárias de $O$. volvulus em biópsias de pele de várias partes do corpo de Raimundo. No quadro II estão os resultados das dissecações de Simulium sp. A. repletas, coletadas uma vez em Raimundo e duas vezes em Roberto. Os insetos não atacaram o pescoço e os pés. $\mathrm{Na}$ análise dos resultados não fo! feita distinção entre as metades direita e esquerda das diferentes regiōes do corpo, combinando-se os resultados dos dipteros coletados no peito e abdome, e nas partes superior e inferior das costas, devido ao número de espécimes coletado. Foi calculada para cada reqião do corpo a média geométrica $\left(\mathrm{M}_{\mathrm{G}}\right)$ se- 
gundo Williams (Leon \& Duke, 1966) de microfilárias de 0 . volvulus. Quando houve 5 mosquitos coletados em uma determinada área do corpo, não foi calculada a média geométrica, sendo apresentado no quadro o número de microfilárias encontradas em cada mosquito. Os resultados acima mostram que em ambos os índios as microfilárias de $O$. volvulus estavam em maior densidade no tronco que nos membros.

\section{QUADRO I}

Distribuição de microfilárias de $O$. volvulus nas diferentes áreas do corpo de Raimundo

\begin{tabular}{l|l|l}
\hline \multicolumn{1}{c|}{ Áreas do corpo } & Biopsios & \multicolumn{1}{c}{ Microfilórios } \\
\hline Pescoço & 1 & 77 \\
Costas & 5 & $37,111,20,8,88$ \\
Nádegas & 2 & 1,1 \\
Peito & 2 & 52,19 \\
Ventre & 2 & 96,2 \\
Braços & 4 & $64,1,16,25$ \\
Antebraços & 4 & $20,6,15,0$ \\
Coxas & 4 & $6,7,12,1$ \\
Pernas & 3 & $25,3,5$ \\
& & \\
\hline
\end{tabular}

QUADRO II

Microfilárias de $O$. volvulus ingeridas por Simulium sp.A em diferentes regĩos do corpo

\begin{tabular}{|c|c|c|c|}
\hline \multirow{2}{*}{ Local de picada } & \multirow{2}{*}{$\begin{array}{l}N .0^{\circ} \text { de } \\
\text { insetos } \\
\text { dissecodos }\end{array}$} & \multicolumn{2}{|c|}{ Microfilários ingeridas } \\
\hline & & (Varioçāo) & $\left(M_{G}\right)$ \\
\hline \multicolumn{4}{|l|}{ ROBERTO } \\
\hline Costas & 22 & $0-201$ & 58.2 \\
\hline Nádegas & 7 & $12-156$ & 58.4 \\
\hline Coxas & 7 & $7-129$ & 30.0 \\
\hline Braços & 20 & $2-450$ & 28.8 \\
\hline Antebraços & 2 & - & $21,230^{\circ}$ \\
\hline Pernas & 2 & - & $0,9^{\bullet}$ \\
\hline Peito & 2 & - & $0,5^{*}$ \\
\hline \multicolumn{4}{|l|}{ RAIMUNDO } \\
\hline Costas & 22 & $4-372$ & 56.3 \\
\hline Nádegas & 3 & - & $1,19,26^{\circ}$ \\
\hline Coxas & 13 & $0-120$ & 8.6 \\
\hline Braços & 9 & $3-171$ & 24.6 \\
\hline Antebraços & 1 & - & $76^{\circ}$ \\
\hline Pernas & 2 & - & $6,24^{*}$ \\
\hline Peito & 3 & - & $38,57,511^{\bullet}$ \\
\hline
\end{tabular}

(*) Contogem direta.
ÍNDICE DE INFECÇÃO EXPERIMENTAL

Os resultados da infecção experimental de Simulium sp. A. alimentados em portadores de $O$. volvulus estão no quadro III. Os insetos não sobreviveram mais do quc 84 horas, morrendo a maioria 36 horas após o repasto infetante. Todos os exemplares recusaram outra refeição sanguínea e aparentemente não se alimentaram da solução açucarada ou da papa de banana. Um grande número de microfilárias era facilmente evidericiado no conteúdo estomacal dos mosquitos mortos até 36 horas após o repasto. Muitas destas microfilárias estavam quebradas, provavelmente devido à ação dos fortes dentes do cibário e da maceração do sangue no processo de preparação. As microfilárias encontradas no conteúdo estomacal geralmente coram-se mal.

Dos 241 dipteros originalmente infetados, $18,2 \%$ (44) apresentavam microfilárias ou larvas salsichóides nos músculos torácicos. Apenas 3 mosquitos apresentaram dois estágios larvários nos músculos, mas morreram 24 horas após o repasto infectante. Do número total de exemplares dissecados, $24.1 \%$ (58) apresentaram microfilárias livres no tórax. O máximo de microfilárias e larvas salsichóides encontradas nos músculos de um só exemplar foi de 8 e 9 , respectivamente. Apenas uma larva salsichóide com intestino em desenvolvimento foi encontrada nos músculos de um exemplar que morreu 60 horas após a infecçăo. No transcurso das primeiras 24 horas após o repasto infetante foi grande o número de insetos encontrados com microfilárias no conteúdo estomacal (quadro II). Em 36 horas houve um decréscimo considerável destas formas e depois de 48 horas tornaram-se progressivamente mais raras até não serem mais detectáveis no conteúdo estomacal.

No quadro IV estão lançados os números de dípteros positivos tanto para microfilárias como para larvas salsichóides na musculatura torácica em relação à ingestão teórica de microfilárias pelos insetos que morreram até 24 horas após a ingestão de sangue infectante, antes que pudesse ocorrer a digestão e excreção de microfilárias. A ingestão inicial é calculada pela soma dos parasitos em qual- 


\section{QUADRO III}

Infecção experimental de Simulium sp. A. com microfilárias de $O$, volvulus

\begin{tabular}{|c|c|c|c|c|c|c|}
\hline $\begin{array}{l}\text { Horas após } \\
\text { infeç̧äo }\end{array}$ & $\begin{array}{l}\text { Insetos } \\
\text { dissecados }\end{array}$ & $\begin{array}{c}\text { Insetos com } \\
\text { mfs no } \\
\text { abdomen }(\%)\end{array}$ & $\begin{array}{l}\text { Insetes com } \\
\text { mfs livre no } \\
\text { torax }(\%)\end{array}$ & $\begin{array}{l}\text { Insets com mfs } \\
\text { nos músculos } \\
\text { torácicos }(\%)\end{array}$ & $\begin{array}{c}\text { Insetos c/ larvas } \\
\text { solsichóides } \\
\text { nos mússulos } \\
\text { torácicos (\%) }\end{array}$ & $\begin{array}{l}\text { Insetos com mfs } \\
\text { e larvas } \\
\text { salsichóides } \\
\text { nos músculos } \\
\text { torócicos }(6\end{array}$ \\
\hline 12 horas & 90 & $72(80)$ & $25(27.8)$ & $10(11.1)$ & $7(7.8)$ & $14(15.5)$ \\
\hline 24 horas & 32 & $30(93.7)$ & $9(28.1)$ & $2(6.2)$ & $4(12.5)$ & $6(18.7)$ \\
\hline 36 horas & 67 & $46(68.7)$ & $21(31.3)$ & $3(4.5)$ & $8(11,9)$ & $11(16.4)$ \\
\hline 48 horas & 31 & $9(29.0)$ & $3(9.7)$ & $O(0)$ & $4(12.9)$ & $4(12.9)$ \\
\hline 60 horas & 16 & $2(12.5)$ & $O(0)$ & $0(0)$ & $4(25.0)$ & $4(25.0)$ \\
\hline 72 horas & 3 & $O(0)$ & $O(0)$ & $O(0)$ & $O(0)$ & $O(0)$ \\
\hline 84 horas & 2 & $0(0)$ & $O(0)$ & $0(0)$ & $2(100.0)$ & $2(100.0)$ \\
\hline TOTAIS & 241 & $159(65.9)$ & $58(24,1)$ & $15(6.2)$ & $29(12.0)$ & $44(18.2)$ \\
\hline
\end{tabular}

quer estágio encontrado por dípteros. Nos casos em que teoricamente era ingerida uma quantidade menor que 20 microfilárias somente uma pequena percentagem apresentou invasão dos músculos torácicos. Nos casos de maiores quantidades ingeridas a invasão era significativamente maior.

Dos simulídeos coletados entre $16,00 \mathrm{e}$ 18,00 horas nos dias 16 e $18 / 12 / 75$ para as cbservações sobre a incidência de ataque, 278

\section{QUADRO IV}

Invasão dos músculos torácicos de Simulium sp. A por microfilárias de $O$. volvulus em relação ao número de microfilárias ingeridas

\begin{tabular}{|c|c|c|c|}
\hline $\begin{array}{l}\text { Ingestāo } \\
\text { inicioi de } \\
\text { mfs }\end{array}$ & $\begin{array}{c}\text { Insetos } \\
\text { dissecados }\end{array}$ & $\begin{array}{l}\text { Insetos com } \\
\text { nifs ou larvas } \\
\text { solsichóides } \\
\text { nos músculos }\end{array}$ & $\begin{array}{l}\% \text { de insetos } \\
\text { com mfs } \\
\text { ou larvas } \\
\text { salsichóides }\end{array}$ \\
\hline $0-10$ & 63 & 4 & 6.3 \\
\hline $11-20$ & 18 & 1 & 5.5 \\
\hline $21-30$ & 11 & 3 & 27.3 \\
\hline $31-40$ & 9 & 3 & 33.3 \\
\hline $41-50$ & 4 & 2 & 50.0 \\
\hline $51-60$ & 6 & 2 & 33.3 \\
\hline Acima de 61 & 11 & 5 & 45.5 \\
\hline Totais & 122 & 20 & 16.4 \\
\hline
\end{tabular}

deles foram dissecados para a procura de $\mathrm{fi}$. iárias, encontrando-se apenas um entre eles com larva salsichóide. A diferença entre a infecção experimental $(18,2 \%)$ e a infecção natural $(0.4 \%)$ foi significante $\left(_{1}^{2}-50, P=0,1\right)$. podendo-se concluir que as microfilárias e larvas salsichóides encontradas na musculatura torácica dos dípteros experimentalmente infetados foram consequeência do repasto sanguineo.

\section{INCIDÊNCIA DE ATAQUE}

Durante os três dias de coletas, foram capturadas 2852 espécimes de Simulium sp. A, três de sp. $B$ e 1 de sp. $C$ atacando o homem. Apenas Simulium sp. A foi coletado em número suficiente para se poder avaliar a incidência de ataque. Como o padrão de picada foi o mesmo, reuniram-se os dados obtidos nos três dias de coletas (quadro V). Cada resultado de captura de meia hora foi expresso pela média de Williams (M ) e em percentagem do total de coletas diárias. Os resultados sobre incidência de ataque são apresentados na fig. 1. $O$ ataque começou entre 06,30 e 07,00 horas de manhã e gradualmente foi aumentando até formar um pico entre 10,00 e 10,30 horas havendo uma estabilização dos ataques até às 13,00 horas. Depois desta hora houve 


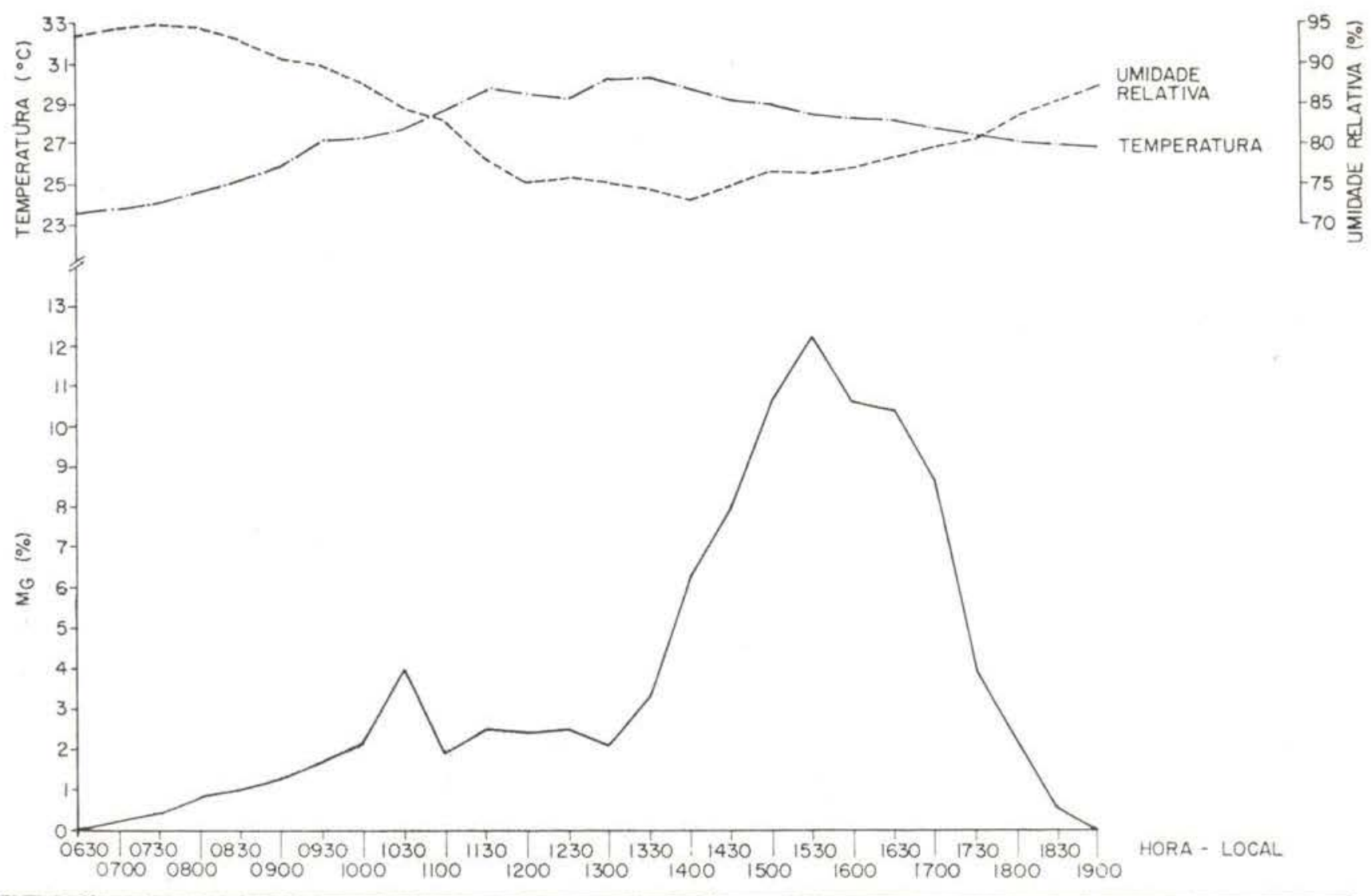

Fig. 1 - Atividade hematófaga (biting cycle) de Simulium sp. A em Toototobi.

uma grande ativaçăo das picadas, sendo o máximo atingido às 15,30 horas. $O$ ataque continuou intenso até às 17,00 horas, decrescendo rapidamente depois e cessando um pouco antes das 18,30 horas.

Durante os três dias de coletas, as manhãs apresentavam-se com grande nebulosidade, que foi diminuindo até ao meio dia, quando o céu apresentava nuvens esparsas. Em dois dias ocorreram leves pancadas de chuvas por períodos curtos, aparentemente sem efeitos sobre a incidência de ataque.

\section{DISCUSSÃo}

Diante dos dados obtidos pode-se ter apenas uma idéia aproximada da distribuição de microfilárias de $O$. volvulus nos dois índios Yanomama.

- Aplicando-se a técnica de Leon \& Duke (1966), ou seja, deixando o Simulium sp. A picar diferentes áreas do corpo, observou-se uma grande variação no número de microfilárias ingeridas por inseto, devido provavelmente, à grande dispersão de picadas em uma determinada área corpórea. Pode ser explicada do mesmo modo a grande variação do número de parasitos encontrados nas biópsias de pele, mesmo levando em consideração o fato dos fragmentos de pele não terem sido pesados. Usando-se os dois métodos para a avaliação da densidade de microfilárias na mesma região do corpo do mesmo índio, quase sempre o número de larvas obtidas foi maior no Simulium sp.A do que nas biópsias de pele. É possível que, ao se alimentar, esta espécie Exerça algum efeito sobre a concentração de microfilárias da pele do hospedeiro, como assinalaram Leon \& Duke (1966) nos simulídeos guatemaltecos. Diante desta interação entre simulídeos e microfilárias, este seria o métodos de escolha na detecção de infecções de baixa densidade, em lugar da tradicional biópsia de pele. 
QUADRO V

Frequências de picadas em homem por Simulium sp. A no rio Toototobi

\begin{tabular}{|c|c|c|c|c|c|c|}
\hline \multirow[b]{2}{*}{ HORA LOCAL } & \multicolumn{3}{|c|}{ INSETOS COLETADOS } & \multirow{2}{*}{$\begin{array}{l}\text { TOTAL } \\
\text { INSETOS } \\
\text { COLLETADOS }\end{array}$} & \multirow{2}{*}{$M_{G}$} & \multirow{2}{*}{$\%^{M}{ }_{G}$} \\
\hline & $16 / 12 / 75$ & $17 / 12 / 75$ & $18 / 12 / 75$ & & & \\
\hline 06,30 & 0 & 0 & 0 & 0 & - & - \\
\hline 07,00 & 7 & 0 & 2 & 9 & 1.9 & 0.2 \\
\hline 07,30 & 7 & 1 & 5 & 13 & 3.6 & 0.4 \\
\hline 08,00 & 15 & 4 & 6 & 25 & 7.2 & 0.8 \\
\hline 08,30 & 26 & 2 & 9 & 37 & 8.3 & 1io \\
\hline 09,00 & 16 & 7 & 13 & 36 & 11.4 & 1.3 \\
\hline 09,30 & 22 & 13 & 10 & 45 & 14.7 & 1.7 \\
\hline 10,00 & 38 & 11 & 16 & 65 & 18.8 & 2.2 \\
\hline 10,30 & 46 & 26 & 34 & 106 & 34.4 & 4.0 \\
\hline 11,00 & 19 & 18 & 12 & 49 & 16.0 & 1.9 \\
\hline 11,30 & 16 & 9 & 60 & 85 & 21.6 & 2.5 \\
\hline 12,00 & 26 & 13 & 25 & 64 & 20.4 & 2.4 \\
\hline 12,30 & 36 & 19 & 14 & 69 & 21.3 & 2.5 \\
\hline 13,00 & 34 & 6 & 26 & 66 & 17.8 & 2.1 \\
\hline 13,30 & 34 & 23 & 29 & 86 & 28.3 & 3.3 \\
\hline 14,00 & 60 & 30 & 89 & 179 & 54.4 & 6.3 \\
\hline 14,30 & 108 & 53 & 56 & 217 & 68.5 & 8.0 \\
\hline 15,00 & 126 & 95 & 65 & 286 & 92.0 & 10.7 \\
\hline 15,30 & 169 & 98 & 68 & 335 & 104.1 & 12.2 \\
\hline 16,00 & 219 & 73 & 47 & 339 & 91.0 & 10.6 \\
\hline 16,30 & 149 & 90 & 53 & 292 & 89.3 & 10.4 \\
\hline 17,00 & 113 & 107 & 34 & 254 & 74.3 & 8.7 \\
\hline 17,30 & 59 & 49 & 13 & 121 & 33.8 & 3.9 \\
\hline 18,00 & 26 & 22 & 12 & 60 & 19.0 & 2.2 \\
\hline 18,30 & 9 & 3 & 2 & 14 & 3.9 & 0.5 \\
\hline 19,00 & 0 & 0 & 0 & 0 & - & - \\
\hline TOTAIS & 1380 & 772 & 700 & 2852 & 8562 & 99.8 \\
\hline
\end{tabular}

Rassi et al. (1975), após seus estudos em maio e junho de 1974 apontam o $S$. amazonicum Goeldi, 1905, como o vetor de $O$. volvulus na regiāo do Toototobi. Consideremos esta afirmativa prematura se baseada nas provas apresentadas. Em primeiro lugar, não estamos totalmente convencidos de que a identificação dos insetos feita por eles esteja correta. A descrição das fêmeas de $S$. amazonicum dadas por Rassi (1974) corresponde em todos os detalhes às fêmeas de Simulium sp. A encontradas em nosso levantamento. Muito embora a genitália feminina do Simulium sp. A seja semelhanter à do $S$. amazonicum descritas do cótipo por Smart (1942), o desenho do escudo figurado por Porto (1939) e confirmado por Smart (1942) como sendo de S. amazo. nicum difere do Simulium sp. A. Como constatamos que o escudo de Simulium sp. A é muito semelhante ao de $S$. haematopotum Malloch 1914, e a genitália femininina difere desta espécie, preferimos chamar de Simulium sp. A à espécie antropófila mais comum no rio Toototobi, até que possamos examinar um cótipo de S. amazonicum Gceldi, 1905. Em segundo lugar, Rassi et al. (1975) pressupõem que as larvas salsichóides por eles obtidas de 7 dos 922 "S. amazonicum" capturados no Toototobi eram de $O$. volvulus, desde que demonstraram que não existe $M$. ozzardi na área. Os macacos da região amazônica e quase certamente os de Toototobi são encontrados co- 
mumente com microfilárias no sangue e, como ainda não foram realizados estudos sobre as preferências alimentares de Simulium sp. A no Toototobi, não se pode garantir que a espécie seja apenas antropófila ou também vetora de filárias de macacos. Ao que sabemos não há uma técnica apropriada para se fazer a distinção com segurança das larvas salsichóides das diferentes espécies de filárias.

Diante dos ciados obtidos fica bem evidenciado que, ao se alimentar, o Simulium sp. A ingere imediatamente as microfilárias de $O$. volvulus e é suscetível à penetração destas microfilárias na musculatura torácica, seguindo-se o desenvolvimento do parasito até o estágio salsichóide, 48 horas após o repasto sanguíneo infectante. Este fato, associado ao grande número de Simulium sp. A picando o homem em Toototobi, sugere que esta espécie é um possivel vetor de oncocercose. Contudo, seu desempenho como vetor só poderá ser confirmado quando fêmeas da Simulium sp. A forem encontradas naturalmente albergando larvas infectantes de $O$. volvulus e quando esta forma do parasito for obtida de simulídeos experimentalmente infetados. Notou-se que era maior a probabilidade de invasão do torax de Simulium sp. A quando havia ingestăo de mais de 20 microfilárias de $O$. volvulus, embora não se pudesse determinar o efeito de uma ingestão maciça sobre a longevidade dos simulídeos. Achado semelhante foi feito por Leon \& Duke (1966) com S. ochraceum, o vetor mais eficiente de oncocercose na Guatemala. Para avaliar a ingestão teórica de microfilárias pelo vetor, Leon \& Duke usaram a média geométrica de microfilárias na área corpórea onde os insetos estavam se alimentando. Não pudemos efetuar a avaliação usando o mesmo método devido à grande variação do número de microfilárias em uma determinada área de pele, substituindo-a pela contagem direta dos parasitos em cada inseto, considerando que todas as larvas salsichóides originaram-se das microfilárias sugadas durante o repasto sanguíneo. Tal determinação de ingestão de microfilárias é apenas aproximada, tanto pelos dificuldades de obtençăo de macerados homogêneos de sangue retirado do estômago de espécimes preservados em ál- cool, quanto pelas possíveis perdas naturais por excreção. Numa amostra pequena como esta, é provável que a contagem direta forneça dados mais exatos do que os obtidos pela média de microfilárias por área de pele.

\section{AgRADECIMENTOS}

Desejamos agradecer aos Srs. Waldomiro Santos de Albuquerque, Benedito Faustino da Silva e Eduardo Vieira da Silva pela assistência técnica, ao Sr. e Sra. B. Hartman pelas condições de trabaiho na missão Toototobi e ao Professor W. L. Paraense pelas facilidades de trabalho na Universidade de Brasilia.

$\mathrm{O}$ trabalho foi realizado com a ajuda financeira do Conselho Nacional de Desenvolvimento Técnico e Científico, Programa do Trópico Ümido.

\section{Summary}

Preliminary studies on various factors influencing the transmission of onchocerciasis were made in December 1975 on the river Toototobi in northern Amazonas. Microfilariae of $\mathbf{O}$. volvulus were more abundant in the trunk than in the limbs of two Yanomama indian volunteers. Three anthrophilic species of Simuliidae were captured and have been temporarily nemaed as Simulium sp.A., sp.B., sp.C. until a definitive identification is made. Only the former species was sbundant biting mainly in the early afternoon. The finding of sausage larva in a naturally infected fly and the successful experimental infection of wild flies with microfilariae of $\mathbf{O}$. volvulus, which subsequently developed to the late sausage stage, suggest that Simulium sp.A is a probable vector of onchocerciasis in the area.

\section{BIBLIOGRAFLA CITADA}

BeArzoti, P., LANe, E. \& Menezes, F., J.

1967 - Relato de um caso de oncocercose adquirida no Brasil. Rev. Paul. Med., 70 : 102 .

DUKE, B.O.L.

1962 - A standard method of assessing microfilarial densities on onchocerciasis surveys. Bull. Wld. Hlth. Org., 27(4-5) : 629-632.

LEON, J.R. DE \& DUKE, B.O.L

1966 - Experimental studies on the transmission of Guatemalan and West African 
strains of Onchocerca volvulus by $\mathbf{S i}$ mulium ochraceum, S. metallicum and S. callidum. Trans. R. Soc. Trop. Med. Hyg., 60(6) : 735-752.

Morats, M. \& Dlas, L.B.

1972 - Oncocercose no Estado do Amazonas, Brasil. Rev. Inst. Med. Trop. São Paulo, $14(5): 330-333$.

Moraes, M.A.P., Fratha H. \& Chaves, G.M.

1973 - Onchocerciasis in Brazil. Bull. Panam. Hith. Org., 7(4) : 50-56.

MoRaes, M.A.P. \& Chaves, G.M.

$1974 \mathrm{a}$ - Um caso de oncocercose no Território de Roraima, Brasil. Rev. Inst. Med. Trop. São Paulo, 16(2) : 110-113.

$1974 \mathrm{~b}$ - Onchocerciasis in Brazil; new findings among the Yanomama indians. Bull. Panam. Hith. Org., 8(2) : 95-99.
PORTO, C.E.

1939 - Simulídeos da região neotrópicn. (II Genero Simulium). Bol. Biol. (N.S.), $4(3): 369-373$

RAssI, E.

1974 - Assessoria para a pesquisa e o controle de oncocercose no Brasil. PAHO, WHO (Brasil-1000D) 1-34. Documento nāo publicado.

Rassi, E.; Lacerda, N.; Guimarães, J.A.; Vulcano, M.A.; RAMirez PÉres, J. \& RAMtrez, A.

1975 - Informe preliminar sobre un nuevo vector de la oncocercosis en las americas: el Simulium amazonicum (Goeldi, Lutz, 1910 y 1917). Bol. Ofic. Sanit. Panam., 79(2) : 136-138.

SMART, J

1942 - Notes on Simuliidae (Diptera). Proc. R. Ent. Soc. Lond. (B), 11(3) . 46-50 